\title{
Cuidados em saúde mental ofertados a profissionais de saúde durante a pandemia de Covid-19
}

\author{
Mental health care offered to health professionals during the Covid-19 pandemic \\ Atención de salud mental ofrecida a los profesionales de la salud durante la \\ pandemia de Covid-19
}

\begin{abstract}
Jocieli Ferrari $\mathbb{D}^{a}$, Priscila Goergen Brust-Renck $\mathbb{D}^{\mathrm{b}}$
a Universidade do Vale do Taquari (UNIVATES), Curso de Psicologia - Lajeado/RS - Brasil. Universidade do Vale do Rio dos Sinos (UNISINOS), Programa de Pós-Graduação em Psicologia Clínica - São Leopoldo/RS - Brasil. b Universidade do Vale do Rio dos Sinos (UNISINOS), Programa de Pós-Graduação em Psicologia Clínica - São Leopoldo/RS - Brasil.
\end{abstract}

Instituição: Universidade do Vale do Rio dos Sinos (UNISINOS), Programa de Pós-Graduação em Psicologia Clínica - São Leopoldo/RS - Brasil

DOI 10.5935/2318-0404.20210010

\section{Resumo}

Este artigo traz aspectos relacionados à atenção à saúde mental dos trabalhadores da saúde da linha de frente durante a pandemia da Doença Coronavírus-2019. O estudo qualitativo teve como objetivo compreender as ações de cuidado em saúde mental ofertadas aos trabalhadores da saúde pública diretamente atuantes no combate à doença e descrever as implicações emocionais que os profissionais de saúde mental experienciaram no trabalho desenvolvido. No município de Lajeado/RS, os profissionais da linha de frente receberam apoio psicossocial e psicoterapia breve por sete psicólogas voluntárias que relataram sua percepção sobre a saúde mental desses profissionais e os desafios do atendimento presencial. Trechos das entrevistas foram utilizados para ilustrar os resultados, a partir das categorias analisadas à luz da análise de conteúdo. Os resultados foram classificados em três categorias: o estado de saúde mental dos trabalhadores de linha de frente, apoio oferecido a esses profissionais, e apoio recebido pelos profissionais de saúde mental para a realização dessas atividades. A análise indicou que muitos profissionais, tanto da linha de frente quanto da saúde mental, referiram sintomas psicológicos desencadeados ou agravados pela pandemia, preocupações com a possibilidade de contaminar alguém próximo, invisibilidade das necessidades em saúde mental da população, e a falta de investimento nesse setor. 
Palavras-chaves: Infecções por Coronavírus; Saúde Mental; Pessoal de Saúde; Sistemas de Apoio Psicossocial; Psicoterapia Breve

\begin{abstract}
This article presents aspects related to the mental health care of frontline health workers during the 2019 Coronavirus Disease pandemic. The qualitative study aimed to understand the mental health care actions offered to public health workers directly active in combating the disease and to describe the emotional implications that mental health professionals experienced in the work developed. In the municipality of Lajeado/RS, frontline professionals received psychosocial support and brief psychotherapy by seven volunteer psychologists who reported their perception of the mental health of these professionals and the challenges of face-to-face care. Excerpts from the interviews were used to illustrate the results from the categories analyzed in the light of the content analysis. The results were classified into three categories: mental health of frontline workers, support offered to these professionals, and support received by mental health professionals to carry out these activities. The analysis indicated that many professionals, both from the front line and from mental health, referred to psychological symptoms triggered or aggravated by the pandemic, concerns about the possibility of contaminating someone close to them, invisibility of the population's mental health needs, and the lack of investment in this. sector.
\end{abstract}

Keywords: Coronavírus Infections; Mental Health; Health Personnel; Psychosocial Support Systems; Psychotherapy Brief

\title{
Resumen
}

Este artículo presenta aspectos relacionados con la atención de la salud mental de los trabajadores de la salud de primera línea durante la pandemia de la enfermedad por coronavirus de 2019. El estudio cualitativo tuvo como objetivo comprender las acciones de atención en salud mental que se ofrecen a los trabajadores de salud pública directamente activos en la lucha contra la enfermedad y describir las implicaciones emocionales que los profesionales de la salud mental experimentaron en el trabajo desarrollado. En el municipio de Lajeado/ RS, los profesionales de primera línea recibieron apoyo psicosocial y psicoterapia breve por parte de siete psicólogos voluntarios que informaron su percepción sobre la salud mental de estos profesionales y los desafíos de la atención presencial. Se utilizaron extractos de las entrevistas para ilustrar los resultados de las categorías analizadas a la luz del análisis de contenido. Los resultados se clasificaron en tres categorías: salud mental de los trabajadores de primera línea, apoyo ofrecido a estos profesionales y apoyo recibido por los profesionales de la salud mental para llevar a cabo estas actividades. El análisis indicó que muchos profesionales, tanto de primera línea como de salud mental, refirieron síntomas psicológicos desencadenados o agravados por la pandemia, inquietudes sobre la posibilidad de contaminar a alguien cercano, invisibilidad de las necesidades de salud mental de la población, y la carencia de inversión en este sector. 
Palabras clave: Infecciones por Coronavirus; Salud Mental; Personal de Salud; Sistemas de Apoyo Psicosocial; Psicoterapia Breve

\section{Introdução}

Efeitos da Doença Coronavírus-2019 (COVID-19) na vida dos profissionais de saúde de linha de frente podem ser identificados em diversos contextos, a partir das implicações emocionais relacionadas ao fato de vivenciar medo e insegurança ao lidar com uma doença silenciosa e sem precedentes ${ }^{1}$. O medo também aparece associado à possibilidade de infecção de pessoas próximas, acarretando a necessidade de mudanças específicas na vida destes profissionais, que restringem ainda mais seu círculo social e afastam-se do convívio familiar, em comparação a outros profissionais durante a pandemia. Essas mudanças ocorridas em suas vidas foram exploradas no âmbito da saúde mental, tendo como base o momento vivenciado no contexto da atual pandemia.

Diversos fatores podem contribuir para o desenvolvimento de sintomas psicopatológicos, uma vez que a experiência negativa é vivida de maneira individual e pode ser provocada por eventos potencialmente estressores ${ }^{2}$. No cenário da pandemia, o desenvolvimento de sintomas negativos tem efeito de grandes proporções nos serviços de saúde onde os profissionais atuam. Relacionado a isso, estudos apontam para a prevalência de sintomas de depressão, ansiedade, insônia e angústia, associados às situações de alto risco que o trabalho nas unidades de saúde tem imposto ${ }^{3}$.

A esse respeito, ações foram desenvolvidas no país com o intuito de ofertar apoio à categoria dos profissionais de saúde. Como exemplo, pode-se citar os call centers criados para realizar atendimento psicológico e psicossocial de maneira remota, com intervenções voltadas ao manejo de crises, psicoeducação sobre sintomas psicológicos que podem ser apresentados, bem como estratégias de enfrentamento e autocuidado. Em grande parte, as intervenções psicossociais e de caráter preventivo se deram de forma breve e, em sua maioria, de modo online $e^{4}$.

O Brasil, ao lado de outros quatro países da América do Sul, ocupa as mais elevadas posições no ranking de incapacidade devido à depressão, classificando os transtornos de ansiedade como a segunda maior incidência ${ }^{5}$. Essa preocupação é pertinente, uma vez que o risco de transmissão nesse público é grande, em razão da alta transmissibilidade do vírus e sendo esta a categoria considerada como a mais exposta ao risco de contaminação da doença'. Os resultados de uma pesquisa realizada nos meses de janeiro e fevereiro de 2020, na China, apontaram que atuar diretamente no combate à COVID-19 aumentava os níveis de ansiedade e estresse, além de apresentar efeitos negativos sobre o sentimento de autoeficácia no trabalho, na qualidade do sono dos profissionais e sobre o apoio social ${ }^{6}$.

Embora sejam necessárias para a segurança da população e o equilíbrio social, ainda há carências no estabelecimento de estratégias para a coordenação dos problemas de saúde, como também dos problemas psicossociais, acarretados pela COVID-197. Ações de apoio psicossocial são colocadas como potenciais 
estratégias, visto que podem estar associadas à redução da probabilidade da população afetada desenvolver sofrimento psíquico ${ }^{8}$. Em consonância a isso, estudos realizados ao longo desse período de pandemia revelaram que o apoio psicossocial e a psicoterapia ofertados às equipes multiprofissionais de saúde, que atuam diretamente com o tratamento de pacientes infectados com a COVID-19, são considerados benéficos ${ }^{9}$.

Os profissionais de saúde mental que prestam assistência em contextos de emergências, como é o caso da pandemia de COVID-19, podem atuar tanto com a população atingida, quanto com os profissionais da linha de frente, por meio de apoio psicossocial e psicoterapia breve. Apoio psicossocial é um termo que descreve um conjunto amplo de ações que tratam de problemas psicológicos, psiquiátricos, e sociais pré-existentes ou aqueles provocados por emergências ou desastres ${ }^{10}$ e que compõe os Primeiros Cuidados Psicológicos (PCP). Estes, são destinados a pessoas que foram expostas a uma situação de crise recentemente e que se encontram em situação de sofrimento"1.

O interesse pelo tema partiu da inserção de uma das autoras no campo da saúde pública, como profissional de saúde mental voluntária, tendo realizado apoio psicológico às equipes multiprofissionais que atuam diretamente no combate à COVID-19. Esta pesquisa buscou a compreensão do cenário loco regional, tomando como esfera um importante foco de transmissão da doença no Estado do Rio Grande do Sul.

Desta forma, buscou-se compreender as ações de cuidado em saúde mental ofertadas aos trabalhadores da saúde pública no contexto da COVID-19, já que poucas intervenções foram relatadas até o momento, bem como as vivências no trabalho voluntário (como ações realizadas, apoio recebido, percepção da saúde mental dos profissionais e condições de trabalho), tendo em vista a realidade experienciada neste município.

\section{Método}

\section{Natureza da pesquisa e amostra}

Foi realizado um estudo qualitativo, para permitir que as participantes relatassem abertamente as experiências de cuidado em saúde mental que vivenciaram no contexto do trabalho, durante a pandemia de COVID-19.

Participaram da pesquisa sete psicólogas, todas mulheres, que realizaram apoio psicossocial e psicoterapia breve de forma voluntária e presencial aos profissionais da linha de frente, em serviços de saúde pública de atenção primária e secundária, do município de Lajeado/RS, durante os primeiros meses da pandemia de COVID-19. Os atendimentos foram realizados de forma individual e grupal e aconteciam uma vez por semana, em dia e horário organizados pela psicóloga do local, conforme sua disponibilidade. Na maioria das unidades, houve maior procura por atendimento individual e os atendimentos grupais aconteciam de forma esporádica, com pelo menos três trabalhadores. Foram assistidos todos os trabalhadores que manifestaram interesse pelo apoio, sendo eles enfermeiros, técnicos de enfermagem, biomédicos, dentistas, auxiliares de saúde bucal, médicos, recepcionistas, farmacêuticos, e auxiliares de limpeza.

Todas as psicólogas foram recrutadas pela Universidade do Vale do Taquari (UNIVATES), localizada no 
referido município, através de uma campanha para atuar de forma voluntária. Das entrevistadas, duas psicólogas eram residentes e atuavam em ESF, duas eram contratadas pela prefeitura, atuando na área da educação e assistência social, duas eram funcionárias da Univates, e outra era psicóloga clínica em consultório particular, no momento da entrevista. Três das participantes contraíram a doença durante o ano de 2020. (A psicóloga que não participou é uma das autoras do presente estudo.) As participantes tinham em média 34,8 anos $(D P=9,1)$ e experiência de atendimento em psicologia clínica que variava entre 2 a 15 anos (Média=6,3 anos; DP=5,4).

Oito psicólogas atuaram como voluntárias, entre o início de abril e o final de junho de 2020, junto às equipes multiprofissionais que trabalham na linha de frente dos serviços de saúde pública do município, de forma presencial. As políticas de atenção primária não preveem psicólogos em suas equipes, portanto, a Universidade do Vale do Taquari (Univates) mobilizou profissionais voluntários, a fim de realizar apoio psicossocial para as equipes multiprofissionais, tanto da atenção primária como secundária, que não contam com estes profissionais no município. Apoio esse que foi realizado de maneira muito distinta das demais ações voltadas ao público citado que, em sua maioria, foram ofertadas de forma remota e sem possibilidade de se conhecer mais aprofundadamente o território e as especificidades da rede de atenção em saúde, assim como as necessidades das equipes de trabalho.

As psicólogas passaram por uma capacitação breve em PCP antes de iniciarem o trabalho, que foi encerrado em função da sua disponibilidade de tempo e do retorno às configurações habituais das equipes de saúde, a partir de certa estabilização no número de casos da COVID-19 e da redução do percentual de ocupação de leitos hospitalares, permitindo a flexibilização das regras de distanciamento social.

\section{Aspectos éticos}

O estudo foi aprovado pela Secretaria Municipal da Saúde de Lajeado/RS e pelo Comitê de Ética em Pesquisa da Universidade do Vale do Rio dos Sinos (CAAE\#37212220.2.0000.5344). Todos os participantes que aceitaram ser entrevistados, assinaram o Termo de Consentimento Livre e Esclarecido.

\section{Coleta e análise dos dados}

Os dados foram coletados entre os meses de setembro e novembro de 2020 , e no mês de fevereiro de 2021, por meio de entrevistas semiestruturadas. A experiência prévia da pesquisadora, como psicóloga voluntária no serviço prestado, foi utilizada para ajudar a organizar o roteiro da entrevista semiestruturada, buscando explorar mais os processos de cuidado aos profissionais de linha de frente e aos próprios profissionais de saúde mental. Três entrevistas foram realizadas de maneira remota, através da plataforma Google Meet e quatro de forma presencial, conforme preferência e disponibilidade das participantes. As entrevistas presenciais ocorreram em local privativo, sugerido pelas entrevistadas, sendo adotados todos os protocolos sanitários vigentes. A duração média das entrevistas foi de 75 minutos.

Foram feitas perguntas abertas para que as participantes relatassem suas percepções em relação (1) à 
saúde mental dos trabalhadores que atuam de forma direta no combate à COVID-19, (2) ao apoio oferecido a esses profissionais, e (3) ao apoio recebido para realização dessas atividades. A pesquisa qualitativa compreende aspectos subjetivos de fenômenos sociais e do comportamento humano, em sua complexidade. Neste método, para que uma análise seja factual, precisa abranger termos da busca qualitativa, trazendo a compreensão da experiência vivenciada a partir do objeto de estudo ${ }^{12}$.

As entrevistas foram gravadas em áudio e transcritas na íntegra para análise posterior. Os dados foram analisados por meio do método de análise de conteúdo, segundo Bardin ${ }^{13}$, seguindo os passos de categorização (codificação), inferência, descrição e interpretação (tratamento das informações). A análise de conteúdo é um conjunto de instrumentos metodológicos, que se aplicam a experiências e significados atribuídos muito diversificados, onde reside uma interpretação controlada e que se baseia na dedução, que é o processo de inferência. Pretende compreender o conteúdo e o continente das experiências, para além de seus significados diretos e imediatos, enriquecendo a visão geral acerca de determinados fatos ${ }^{13}$.

\section{Resultados e discussão}

Os dados coletados são analisados a partir de três categorias, organizadas conforme sua ocorrência e temática, listadas como: (1) o estado de saúde mental dos trabalhadores que atuam de forma direta no combate à COVID-19, (2) apoio oferecido aos profissionais da linha de frente, e (3) apoio recebido pelos profissionais de saúde mental para a realização dessas atividades.

Em relação à percepção da saúde mental dos trabalhadores que atuam de forma direta no combate à COVID-19, a maioria das participantes refere que havia grande preocupação por parte destes profissionais em contaminar alguém de sua família, em razão de estarem em contato com pacientes infectados, o que fez com que muitos se afastassem de seus entes por longos períodos de tempo, exacerbando ainda mais o sofrimento emocional. A literatura sobre esta temática aponta que as equipes têm sofrido pelo risco de contaminar a si e a sua família, visto que enfrentam ou enfrentaram em algum momento a escassez de equipamentos de proteção individual (EPI), que os auxiliam na proteção de sua saúde, e angustiam-se por não receberem informações claras e precisas das autoridades', apesar do conhecimento da doença já obtido desde o início desta situação. Aliás, a demanda e a jornada de trabalho desses profissionais aumentaram significativamente, tendo em vista a sobrecarga dos sistemas de saúde e o agravamento dos casos de infecção, a partir da evolução da pandemia e do aumento no número de $\operatorname{casos}^{14}$.

"Os trabalhadores traziam muita preocupação com a família, de pegar o vírus e levar pra casa e infectar as pessoas." (Participante 4)

"Alguns disseram que pensaram em ficar num hotel ou morar em outro lugar, porque tavam muito preocupados com a família, que não se perdoariam se passassem o vírus pra alguém, mas que não tinham condições financeiras pra fazer isso. Se afastaram de quem deu, como pai, mãe, avós, tios, por bastante tempo." (Participante 6) 
Consoante a isso, as participantes relatam que a maior dificuldade enfrentada pelos profissionais da linha de frente foi o agravamento de situações/sintomas pré-existentes e intensificados pela pandemia, como por exemplo: automutilação, ideação suicida, problemas de relacionamento com a equipe, violência doméstica, conflitos conjugais e uso abusivo de álcool. Grande parte dos profissionais envolvidos diretamente no enfrentamento da pandemia de Covid-19, e que participaram desta intervenção, já apresentava sintomas anteriores ao evento, em sua maioria não tratados ao longo da vida. Vale destacar, também, que algumas equipes foram realocadas para outras unidades, em razão das demandas atendidas em cada local e dos afastamentos provocados por suspeitas ou confirmações de infecção por coronavírus.

"Eu tive que fazer psicoterapia breve com alguns profissionais, porque tiveram situações graves, de sofrimento bem intenso, sabe? Coisas pessoais, que já aconteciam antes da pandemia, mas que ficou muito pior com tudo que estavam vivenciando no trabalho." (Participante 2)

"Algumas equipes exigiram bastante, em termos de atendimento. Tinha casos bem complexos e pessoas que precisavam de muito apoio." (Participante 3 )

Em geral, as equipes multiprofissionais que têm trabalhado diretamente no combate à COVID-19 (i.e., na chamada "linha de frente"), e as equipes de saúde mental que realizam apoio psicossocial a esses profissionais, executam um novo modo de trabalho, como consequência de diversas reestruturações ${ }^{15}$. Essas reestruturações são concernentes aos novos protocolos de tratamento dos pacientes, de utilização de EPI, de manejo da situação de crise, de realocação de pessoal, de fechamento ou ampliação de unidades de saúde e até de reorganização da demanda recebida pelo serviço ${ }^{16}$.

As psicólogas entrevistadas também identificaram nos profissionais de linha de frente sintomas depressivos e ansiosos, de estresse, angústia e sensação de impotência, desencadeados pelo evento da pandemia, especialmente no que se refere à falta de informações precisas das autoridades, como também aos rígidos protocolos de higiene e isolamento. Em geral, situações de crise, que requeiram mudança e reestruturação de vida, podem propiciar o desenvolvimento de sintomas de estresse, por configurarem-se potencialmente traumáticas. Dados da International Labour Organization ${ }^{17}$ referem que reações físicas, mudança no comportamento e no nível de atividade, e condições de saúde mental pré-existentes são apontados como riscos psicossociais que podem aumentar com a pandemia.

"As pessoas ficaram com os nervos mais à flor da pele, com medo, insônia, angústia e buscaram atendimento. Tavam bem cansados com o uso de EPI, com a necessidade de ficar isolados em casa, de só poder ir pro trabalho." (Participante 3)

"Eles (os trabalhadores) tavam confusos em relação a suas rotinas, tava tendo muitos conflitos entre os trabalhadores, de não se entenderem, assim." (Participante 7) 
As iniciativas adotadas em todo o mundo para conter o avanço da COVID-19 são baseadas no Regulamento Sanitário Internacional (RSI), que estabelece conceitos e medidas que devem ser tomadas como resposta aos graves riscos de saúde pública, pela comunidade internacional ${ }^{18}$. A ausência de uma política nacional coordenada para conter a transmissão do vírus e a falta de coesão entre os Estados - onde as medidas de contenção da doença são tomadas de forma isolada, ignorando a necessidade de articulação entre as divisas - denuncia deficiências e fragilidades no planejamento governamental brasileiro o que, consequentemente, afeta o setor da saúde ${ }^{19}$.

Encontram-se estudos, realizados na $\mathrm{China}^{3}$ e na Itália ${ }^{20}$ por exemplo, que apontaram para a prevalência de sintomas de depressão, ansiedade, insônia e angústia, associados às situações de alto risco que o trabalho nas unidades de saúde tem imposto durante a pandemia. Evidências de síndrome de burnout, risco de suicídio, e transtorno de estresse pós-traumático também foram destacadas por esses estudos, quando se trata da saúde mental dos trabalhadores de saúde diretamente atuantes no enfrentamento da COVID-19.

Uma pesquisa realizada no Canadá mostrou um significativo impacto psicológico da doença na população, que apresentou um alto nível de medo, mesmo no início da pandemia ${ }^{21}$. Esse medo parece estar sendo experimentado pela população em geral e pelos profissionais de saúde de todo o mundo, que têm enfrentado incertezas diante de um vírus silencioso, como também descrenças nos sistemas de saúde e nos governos, em relação à preparação para o enfrentamento da pandemia ${ }^{22}$.

Sobre o apoio oferecido aos profissionais da linha de frente, as maiores ocorrências de relatos nas entrevistas dizem respeito à necessidade do apoio às equipes, que estavam em sofrimento e puderam ter um espaço de fala e escuta qualificada no trabalho, sentindo-se apoiados. Muitos sentiram-se acolhidos e seguros por ter suporte e validação dos sentimentos que experimentavam em uma situação de crise, sem precedentes.

"É importante ter esse cuidado sempre, pros trabalhadores da saúde, que sim, tem as suas questões pessoais, mas vivem o tempo todo questões de outras pessoas e, às vezes, não sabem o limite entre o que é do outro e o que é seu, né? E aí adoecem." (Participante 3)

"Acho que teve uma abertura pra olhar pra saúde mental dos trabalhadores. E também teve uma abertura do próprio trabalhador para a sua saúde mental. É um permitir-se, um olhar-se e defender a importância disso." (Participante 5)

Muitas das fragilidades apontadas podem causar sofrimento emocional aos trabalhadores, que já enfrentam dificuldades anteriores à pandemia, no desempenho de sua função, como o subfinanciamento do Sistema Único de Saúde (SUS). Apesar das carências financeiras, o SUS tem se mostrado elemento central no enfrentamento da pandemia, articulando os diversos níveis de atenção que compõem a rede de saúde ${ }^{23}$. As deficiências enfrentadas por aquele que é o maior sistema público do mundo se dão, especialmente, pelo subfinanciamento. Contudo, o SUS é fortalecido pelas equipes que investem na promoção de uma saúde pública de qualidade, o que reforça ainda mais sua significância ${ }^{24}$. 
No entanto, essa abertura para receber apoio não foi geral. As equipes, por vezes, não se colocavam muito abertas às ações de apoio psicossocial, por insegurança ou por incompreensão sobre o papel da psicóloga na equipe; de dificuldade de reunir os trabalhadores para um momento coletivo, como também de grande satisfação em perceber movimentos da equipe no reconhecimento de suas necessidades físicas e emocionais e reivindicar suporte.

"No início o pessoal tava mais resistente, perguntava: uma psicóloga aqui pra que? Veio saber se a gente tá trabalhando direito ou se a gente tá louco? Se alguém vai querer pegar atestado? Depois eles foram percebendo que eu não tava lá pra vigiar o trabalho deles e contar pra chefia, mas que eu tava lá pra ajudar quem tivesse necessidade, quem tava sofrendo." (Participante 1)

"Cada um ficava numa sala. Tinha grupinhos e os grupinhos ficavam alocados em salas e as pessoas não interagiam entre elas. E isso também não era uma coisa que tava fazendo bem, porque elas precisavam trabalhar juntas, né? No começo foi mais difícil, mas depois eu já vi o pessoal fazendo alguns movimentos pra chamar todo mundo." (Participante 7)

Apesar disso, estudos revelaram que o apoio psicológico e psicossocial ofertado às equipes multiprofissionais de saúde, que atuam diretamente com o tratamento de pacientes infectados com a COVID-19, são considerados benéficos ${ }^{9-6}$. Em situações desgastantes, que demandam muito emocionalmente, como no cenário destacado, ter um profissional que seja referência para suporte, o qual a equipe sabe que pode acionar em caso de necessidade, é fundamental para a promoção e prevenção da saúde mental. Ademais, ações de apoio psicossocial podem estar associadas à redução da probabilidade da população afetada desenvolver sofrimento psíquico ${ }^{8}$.

"Então, no final, as pessoas passaram a ficar muito gratas, assim. As pessoas agradeceram muito, queriam muito que continuasse a ter um serviço como esse, que pudessem contar com o apoio sempre. Que passaram por esse momento de uma forma mais tranquila, pelo fato de eu estar ali disponível, de saber que tinha alguém ali por eles, que ia escutar e não ia julgar os sentimentos deles." (Participante 6)

As psicólogas também mencionam que no final do período de atuação delas nas unidades, as equipes manifestaram desejo de ter um profissional psicólogo inserido nestes locais de forma permanente, que possa trabalhar a prevenção de sintomas emocionais, por identificarem a importância desse cuidado. A fala de grande parte dos profissionais carrega a queixa sobre a falta de investimento e reconhecimento da saúde mental no município, o que acentua os prejuízos da invisibilidade dos sintomas psicológicos dos trabalhadores da linha de frente. 
"O que eu percebo, no município assim, é que não tem nada de cuidado de saúde mental pros trabalhadores da saúde." (Participante 3)

"A gente percebe que quando falam de algum problema, que tem a vercom o trabalho até, não se dá a importância devida. Falta um olhar pro sofrimento, pra saúde mental das equipes." (Participante 7)

Dados do relatório sobre a saúde mental nas Américas ${ }^{25}$ alertam para o déficit de financiamento em saúde mental, para a carga subestimada dos transtornos mentais, para a falta de treinamento contínuo e acesso a insumos, para a privação do apoio de líderes e chefias, e a necessidade de vontade política para produzir mudanças significativas nos serviços. As ações realizadas nas unidades de saúde do município proporcionaram certa visibilidade à saúde mental dos trabalhadores, contudo, de forma fugaz, sendo o investimento ainda escasso.

No que tange ao apoio recebido pelos profissionais de saúde mental para a realização do trabalho voluntário, boa parte das participantes relatam que receberam apoio da equipe de supervisão, composta por todas as psicólogas envolvidas na ação, e que acontecia semanalmente, onde eram discutidas as intervenções realizadas e os obstáculos encontrados. As participantes referem a busca de apoio em diversas frentes. A partir disso, com maiores condições emocionais, trabalharam no apoio aos profissionais da linha de frente, auxiliando-os no cuidado de sua saúde mental. A busca de apoio também ocorreu entre colegas de profissão, por meio de em cursos de curta duração, em psicoterapia pessoal, e supervisão individual, bem como na família, ao identificarem limitações pessoais a partir dos reveses de vivenciar uma realidade tão dura e tão próxima daqueles a quem assistiam.

"Eu acabei buscando apoio porque senti que era muita coisa pra dar conta sozinha, estava me sentindo cansada e bastante ansiosa. Tinha momentos em que era difícil separar aquilo que eu vivia do que os trabalhadores estavam vivendo, eu sentia o que eles também estavam sentindo, por isso levei pra minha terapia e conversei com colegas." (Participante 1)

"Acho que era muito bom os momentos de supervisão, poder escutar as colegas, saber que não era só eu que tinha alguma dificuldade, foi bem significativo." (Participante 4)

Para além do emprego de teorias e técnicas, o trabalho do terapeuta é permeado por emoções e comportamentos pessoais, que podem ser evidenciados a partir da relação com o paciente. Nesse sentido, a supervisão exerce papel importante na atuação do terapeuta, ampliando repertórios e desenvolvendo habilidades no contexto terapêutico ${ }^{26}$. Em relação ao cenário da prática realizada, depreende-se que a supervisão coletiva exercida colaborou para as ações propostas, ao assegurar um ambiente continente e reforçador de apoio e cuidado. Além disso, muitas participantes realizaram cursos de capacitação ao longo do período que estiveram nas unidades de saúde, em virtude de diversos cursos terem sido ofertados nos primeiros meses da pandemia, inclusive de forma gratuita. 
Outrossim, mais da metade expõe que experienciou sintomas de ansiedade, cansaço e estresse no contexto da prática pesquisada, evidenciando um olhar também para si, considerando a complexidade do cenário e as emoções envolvidas. Elas indicaram sobrecarga de trabalho, em razão de equipes muito demandantes por atendimento. Diferentemente das equipes da linha de frente, com trabalhadores concursados e/ou contratados no município, as voluntárias tiveram a escolha de proteger-se em casa, no entanto, escolheram trabalhar no cuidado do outro, que apresentava grande necessidade. Essa iniciativa foi muito significativa para todos os profissionais envolvidos, tanto os que receberam, quanto os que prestaram apoio, se destacando como modelo, inclusive, para outros municípios.

"Me senti bem cansada, nos dias que eu ia pras unidades eu ficava esgotada. Percebi que fiquei mais estressada, acabava brigando em casa por motivos bobos, me sentia ansiosa também, querendo dar conta do trabalho e ajudar as pessoas que tavam precisando." (Participante 2)

As profissionais de saúde mental também se deparam com o grande desafio de tratar pacientes e promover ações de prevenção à doença, ao mesmo tempo que precisavam proteger-se e adaptar-se a um novo modo de trabalho. Elas vivenciaram situações e experienciaram sintomas muito semelhantes aos profissionais diretamente atuantes no combate à COVID-19. Estiveram atuando em um trabalho presencial, em contato com diversos trabalhadores e em unidades com grande circulação de pacientes (inclusive sintomáticos e/ou com diagnóstico positivo para COVID-19), necessitando, por isso, afastar-se de familiares e amigos, seguindo protocolos sanitários ainda mais rígidos. Todas elas realizaram mudanças na vida familiar, social e laboral, da mesma forma que a maioria dos trabalhadores da linha de frente também precisou adotar, visando a autoproteção e a proteção coletiva.

"Eu fiquei sem ver minha família por três meses, fiquei totalmente afastada de todos, pelo risco, né? Quando chegava em casa, parecia zona de guerra: tirava toda a roupa, colocava pra lavar e ia direto pro banho." (Participante 4)

Ademais, cabe sublinhar que a metade das participantes avalia que o momento da entrevista foi importante para ponderar o que aconteceu no período vivenciado, salientando que não haviam refletido sobre o sentido de todas as experiências evocadas e o quanto isso também contribui para o processo de elaboração do vivido. Ainda, as participantes avaliam que o trabalho realizado proporcionou a elas grande aprendizado pessoal e profissional, também identificaram a importância de conhecerem a rede de saúde do município e de estarem capacitadas para a realização do trabalho proposto.

"Nossa, acabei revivendo muitos momentos, agora com a entrevista. Ainda não tinha parado pra pensar em tudo que aconteceu, em como me senti. Foi bem importante pensar nisso pra mim." (Participante 6)

"Eu vi esse trabalho como uma oportunidade de crescer, de aprender, pra mim tá 
trabalhando com os trabalhadores, tá dando um apoio tão necessário, porque na unidade que eu tava, eu percebi o quanto esses trabalhadores tavam assustados, né?." (Participante 2)

Compreende-se, nesse sentido, que a entrevista serviu como facilitadora a um processo de elaboração psíquica, no qual ocorre a assimilação das ocorrências internas e externas do sujeito ${ }^{27}$. A apropriação e a representação consciente do conteúdo das entrevistas pode, de certa forma, possibilitar que as participantes pudessem (re)significar o momento experimentado, o que contribui para a redução da probabilidade de desenvolver sofrimento psíquico.

"Parar pra pensar em tudo que aconteceu foi muito bom, apesar de ter sido um momento difícil, sabe? Vivi várias coisas com minha família e ouvi coisas difíceis dos trabalhadores nas unidades. Mas ter essa reflexão agora fez a diferença." (Participante 2)

"Conhecer a rede me fez entender melhor o que eu poderia fazer pelas equipes. Fico pensando como foi pras outras colegas, porque esse conhecimento prévio fez toda a diferença pra mim. E o treinamento que recebemos sobre os primeiros cuidados psicológicos também, ajudou a direcionar as ações." (Participante 5)

\section{Conclusão}

Este estudo possibilitou ampliar a compreensão sobre as ações de cuidado em saúde mental ofertadas aos trabalhadores da saúde pública no contexto da pandemia de COVID-19, além de problematizar as vivências de psicólogas voluntárias na atuação presencial em unidades de saúde. Verificou-se que muitos profissionais, tanto da linha de frente quanto da saúde mental apresentaram sintomas psicológicos desencadeados pelos desafios encontrados em suas práticas, bem como tiveram exacerbados sintomas pré-existentes.

Os profissionais diretamente atuantes no combate à doença enfrentaram (e ainda enfrentam) muitas adversidades em seu cotidiano, distanciando-se da família e amigos, aumentando a jornada de trabalho, colocando-se em risco para cuidar de quem precisa. Destarte, os profissionais de saúde mental também depararam-se com obstáculos e, apesar de serem treinados para assistir aos que enfrentam situações adversas em um cenário específico e emocionalmente distante, se viram afetados por dificuldades muito semelhantes às dos seus pacientes, onde o distanciamento emocional já não era mais possível. Diversas áreas (e.g., profissional, pessoal, social) da vida do profissional de saúde podem ser afetadas nesse momento de pandemia, especialmente em decorrência de sintomas psicológicos negativos. Identificar quais são essas áreas, qual fator tem mais influência e como cada profissional percebe o efeito disso em sua vida é primordial para auxiliá-lo no enfrentamento da situação de emergência, facilitando a adaptação à rotina e a diminuição do sofrimento ${ }^{28}$. 
Os profissionais da linha de frente e do apoio psicossocial, apesar de estarem realizando um meritório trabalho na saúde pública, têm enfrentado muitas aflições em seu cotidiano ${ }^{29}$. A literatura encontrada nessa temática não realiza subdivisão entre estas duas categorias profissionais, trazendo aspectos comuns aos profissionais de saúde de maneira geral, destacando em grande parte, porém, os profissionais da linha de frente ${ }^{30-31}$. No entanto, apesar da globalização dos efeitos psicológicos apontados, algumas diferenças podem ser encontradas em determinados grupos de profissionais, a partir das especificidades de cada classe e das estratégias de enfrentamento (coping) adotadas por cada profissional| ${ }^{32}$. Os sintomas apresentados pelos profissionais são o ponto de partida para a busca de estratégias de enfrentamento. Permitir-se sentir, procurar suporte e compreensão dos sintomas emocionais auxilia na realização do trabalho a que se propõem.

Os fatores psicossociais relacionados ao sofrimento no trabalho, aliados à falta de investimento na saúde mental, têm agravado muitas situações, especialmente aos profissionais da saúde pública que, no cenário de uma pandemia, vivem as implicações emocionais do trabalho de forma intensa, em razão de serem a referência no cuidado em saúde da população. Esses dados demonstram a valia de ações voltadas à saúde mental daqueles que enfrentam sofrimento em seu cotidiano, e a necessidade de implementação de medidas de prevenção e tratamento nessa esfera, para o fortalecimento dos sistemas de saúde. Ressalta-se a urgência do desenvolvimento de estratégias de promoção e prevenção da saúde mental dos trabalhadores de saúde, que são o mote propulsor na defesa da vida da população, especialmente em tempos emergenciais, como o de uma pandemia.

O estudo realizado apresentou limitações quanto ao tamanho da amostra que, ao se apresentar em número reduzido, permite considerar os resultados encontrados apenas para a população em questão. Outra limitação importante foi a população pesquisada, que está inserida em um município com um número pequeno de profissionais de saúde, apesar deste ter apresentado significativa relevância em números de casos de COVID-19 no Estado do Rio Grande do Sul, proporcionalmente ao número de habitantes.

\section{Agradecimento}

O presente trabalho foi realizado com apoio da Coordenação de Aperfeiçoamento de Pessoal de Nível Superior - Brasil (CAPES) - Código de Financiamento 001.

\section{Referências}

1. Ornell F, Halpern SC, Kessler FHP, \& Narvaez JCM. The impact of the COVID-19 pandemic on the mental health of healthcare professionals. Cadernos de Saúde Pública, 2020; 36(4), e00063520. doi:10.1590/0102$311 \times 00063520$

2. Lara PG, Silva GR, Servino LL, Frimm VC, \& Kristensen $C H$. Primeiros socorros psicológicos: Intervenção em crise para eventos de violência urbana. Revista Educar Mais, 2019; 3(3), 9-16. doi:10.15536/ reducarmais.3.2019.9-16.1607

3. Lai J, Ma S, Wang Y, Cai Z, Hu J, Wei N, Wu J, Du H, Chen T, Li R, Tan H, Kang L, Yao L, Huang M, Wang H, 
Wang G, Liu Z, Hu S. Factors associated with mental health outcomes among health care workers exposed to coronavirus disease 2019. JAMA Netw Open, 2020;3(3), e203976. doi:10.1001/jamanetworkopen.2020.3976

4. Teixeira CFS, Soares CM, Souza EA, Lisboa ES, Pinto ICM, Andrade LR, \& Espiridião MA. A saúde dos profissionais de saúde no enfrentamento da pandemia de Covid-19. Ciência \& Saúde Coletiva, 2020; 25(9): 3465-3474. doi:10.1590/1413-81232020259.19562020

5. Organización Panamericana de la Salud. La carga de los trastornos mentales en la Región de las Américas, 2018. Washington, D.C.: OPS, 2018. Recuperado em 12/2020, de https://iris.paho.org/bitstream/ handle/10665.2/49578/9789275320280_spa.pdf?sequence=9\&isAllowed=y

6. Xiao H, Zhang Y, Kong D, Li S, \& Yang N. The effects of social support on sleep quality of medical staff treating patients with coronavirus disease 2019 (COVID-19) in january and february 2020 in China. Medical science monitor: international medical journal of experimental and clinical research, 2020; 26. doi:10.12659/ MSM.923549

7. Serafini G, Parmigiani B, Amerio A, Aguglia A, Sher L, \& Amore M. The psychological impact of COVID-19 on the mental health in the general population. QJM: An International Journal of Medicine, 2020. doi:10.1093/ qjmed/hcaa201

8. Inter-Agency Standing Committee (IASC, Comitê Permanente Interagências). Diretrizes do IASC sobre saúde mental e apoio psicossocial em emergências humanitárias. Tradução de Márcio Gagliato. Genebra: IASC, 2007. Recuperado em 07/2020, em https://interagencystandingcommittee.org/system/files/iasc_mhpss_ guidelines_portuguese.pdf

9. Cipolotti L, Chan E, Murphy P, van Harskamp N, \& Foley JA. Factors contributing to the distress, concerns, and needs of UK Neuroscience health care workers during the COVID-19 pandemic. Psychology and Psychotherapy: Theory, Research and Practice, 2020, e12298. doi:10.1111/papt.12298

10. World Health Organization \& The UN Refugee Agency, 2012. Assessing mental health and psychosocial needs and resources: toolkit for humanitarian settings. Recuperado em 06/2020, de https://apps.who. int/iris/bitstream/handle/10665/76796/9789241548533_eng.pdf

11. Organização Pan-Americana da Saúde. Primeiros cuidados psicológicos: guia para trabalhadores de campo. Brasília, DF: OPAS, 2015.

12. Minayo Maria Cecília de Souza. Análise qualitativa: teoria, passos e fidedignidade. Ciência \& Saúde Coletiva [Internet]. 2012 Mar [citado 2021 Maio 13] ; 17( 3 ): 621-626. Disponível em: http://www.scielo.br/ scielo.php?script=sci_arttext\&pid=S1413-81232012000300007\&lng=pt. https://doi.org/10.1590/S141381232012000300007.

13. Bardin, L. Análise de conteúdo. São Paulo: Edições 70; 2011.

14. Santarone K, McKenney M, \& Elkbuli A. Preserving mental health and resilience in frontline healthcare workers during COVID-19. American Journal of Emergency Medicine, 2020. doi:10.1016/j.ajem.2020.04.030

15. Shigemura, J., Ursano, R. J., Morganstein, J. C., Kurosawa, M., \& Benedek, D. M. Public responses to the novel 2019 coronavirus (2019-nCoV) in Japan: Mental health consequences and target populations. Psychiatry and Clinical Neurosciences, 2020; 74(4), 281-282. doi:10.1111/pcn.12988

16. Ministério da Saúde, 2020. Sistema Único de Saúde (SUS): Estrutura, princípios e como funciona. Recuperado em 06/2020, de https://www.saude.gov.br/sistema-unico-de-saude

17. International Labour Organization. Managing work-related psychosocial risks during the COVID-19 pandemic - Geneva: ILO, 2020. Recuperado em 07/2020, de https://www.ilo.org/global/topics/safety-and-healthat-work/resources-library/publications/WCMS_748638/lang--en/index.ht 
18. World Health Organization. International Health Regulations, 2005; 3a ed. Recuperado em 07/2020, de https://apps.who.int/iris/bitstream/handle/10665/246107/9789241580496-eng.pdf

19. Cimini, F., Julião, N. A., Souza, A., Ferreira, J. V. S., Figueiredo, G. R., Garcia, L. F. G., Hargreaves, R. T., \& Baggia, F. Nota Técnica: Análise das primeiras respostas políticas do Governo Brasileiro para o enfrentamento da COVID-19 disponíveis no Repositório Global Polimap. Centro de Desenvolvimento e Planejamento, UFMG, 2020. Atualizado em 06/05/2020. Recuperado em 07/2020, de https://www.cedeplar.ufmg. $\mathrm{br} /$ noticias/1242-nota-tecnica-analise-das-primeiras-respostas-politicas-do-governo-brasileiro-para-oenfrentamento-da-covid-19-disponiveis-no-repositorio-global-polimap

20 Sani, G., Janiri, D., Di Nicola, M., Janiri, L., Ferretti, S., \& Chieffo, D. Mental health during and after the COVID-19 emergency in Italy. Psychiatry and Clinical Neurosciences, 2020. doi:10.1111/pcn.13004

21. Asmundson, G. J. G., \& Taylor, S. Coronaphobia: Fear and the 2019-nCoV outbreak. Journal of Anxiety Disorders, 2020; 70, e102196. doi:10.1016/j.janxdis.2020.102196

22. Brooks, S. K., Webster, R. K., Smith, L. E., Woodland, L., Wessely, S. W., Greenberg, N., \& Rubin, G. J. The psychological impact of quarantine and how to reduce it: rapid review of the evidence. The Lancet, 2020; 395(10227), 912-920. doi:10.1016/S0140-6736(20)30460-8

23. Fundação Oswaldo Cruz, 2020. SUS, central para enfrentar a pandemia. Centros de Estudos Estratégicos da FIOCRUZ. Pesquisa, Política e Ação em Saúde Pública. Recuperado em 07/2020, de https://cee.fiocruz. $\mathrm{br} /$ ?q=node $/ 1140$

24. Universidade Federal do Rio Grande do Sul, 2020. SUS e os desafios de uma pandemia. Recuperado em 07/2020, de https://www.ufrgs.br/coronavirus/base/entrevista-sus-e-os-desafios-de-uma-pandemia/

25. OPAS/OMS Brasil. Folha informativa - COVID-19 (doença causada pelo novo coronavírus). Recuperado em 06/2020, de https://www.paho.org/bra/index.php?option=com_content\&view=article\&id=6101:covid19 \&ltemid $=875$

26. Sartori, Raquel Martins. O papel da supervisão na formação de terapeutas comportamentais: estudo de caso. Estudos Interdisciplinares em Psicologia, 2014; 5(1), 96-108. https://dx.doi.org/10.5433/22366407.2014v5n1p96

27. Roussillon, René. A elaboração e seus modelos: Leitura principal, Congresso da IPA em Berlim, Julho de 2007. Psicologia USP, 2016; 27(2), 367-374. https://doi.org/10.1590/0103-656420150085

28. Pfefferbaum, B., Flynn, B., Schonfeld, D., Brown, L., Jacobs, G., Dodgen, D., Donato, D., Kaul E. R., Stone, B., Norwood, A. E. , Reissman, D. B., Herrmann, J., Hobfoll, S. E., Jones, R. T., Ruzek J. I. , Ursano, R. J. , Taylor, R. J., \& Lindley, D. The integration of mental and behavioral health into disaster preparedness, response, and recovery. Disaster Medicine and Public Health Preparedness, 2012; 6(1), 60-66. doi:10.1001/dmp.2012.1

29. Holmes, E. A., O'Connor, R. C., Perry, V. H., Tracey, I., Wessely, S., Arseneault, L., Ballard, C., Christensen, H., Silver, R. C., Everall, I., Ford, T., John, A., Kabir, T., King, K., Madan, I., Michie, S., Przybylski, A., Shafran, R., Sweeney, A., Worthman, C. M., Yardley, L., Cowan, C., Cope, C., Hotopf, M., \& Bullmore, E. Multidisciplinary research priorities for the COVID-19 pandemic: a call for action for mental health science. The Lancet Psychiatry, 2020; 7(6), 547-560. doi:10.1016/S2215-0366(20)30168-1

30. Jiang X, Deng L, Zhu Y, Ji H, Tao L, Liu L, Yang D, \& Ji W. Psychological crisis intervention during the outbreak period of new coronavirus pneumonia from experience in Shanghai. Psychiatry Research, 2020; (286), e112903. doi:10.1016/j.psychres.2020.112903

31. Schmidt B, Crepaldi MA, Bolze SDA, Neiva-Silva L \& Demenech LM. Impacts on mental health and psychological interventions related to the new coronavirus pandemic (COVID-19). SciELO - Scientific Electronic Library Online, 2020 [Preprint] doi:10.1590/SciELOPreprints.58 
32. Brooks, S. K., Dunn, R., Amlôt, R., Rubin, J., \& Greenberg, N. Protecting the psychological wellbeing of staff exposed to disaster or emergency at work: A qualitative study. BMC Psychology, 2019; 7(78), 1-11. doi:10.1186/s40359-019-0360-6

Contribuições: Jocieli Ferrari - Análise estatística, Aquisição de financiamento, Coleta de Dados, Conceitualização, Gerenciamento de Recursos, Gerenciamento do Projeto, Investigação, Metodologia, Redação - Preparação do original, Redação - Revisão e Edição - Validação e Visualização;

Priscila Goergen Brust-Renck - Gerenciamento do Projeto, Metodologia, Redação - Preparação do original, Redação - Revisão e Edição - Supervisão e Validação.

\section{Correspondência}

Jocieli Ferrari

joferrari88@gmail.com

Submetido em: 01/02/20201

Aceito em: 05/06/2021 